\title{
Iterative Signal Processing in Communications
}

\author{
Christian Schlegel, ${ }^{1}$ Peter Hoeher, ${ }^{2}$ Owe Axelsson, ${ }^{3}$ and Lance Pérez ${ }^{4}$ \\ ${ }^{1}$ Department of Computing Science, University of Alberta, Edmonton, AB, Canada T6G 2E8 \\ ${ }^{2}$ Faculty of Engineering, Christian-Albrechts-University of Kiel, 24143 Kiel, Germany \\ ${ }^{3}$ Institute of Geonics, AS CR, Ostrava, 60200 Brno, Czech Republic \\ ${ }^{4}$ Department of Electrical Engineering, University of Nebraska-Lincoln, Lincoln, NE 68588, USA
}

Correspondence should be addressed to Christian Schlegel, schlegel@ualberta.ca

Received 22 September 2010; Accepted 22 September 2010

Copyright () 2010 Christian Schlegel et al. This is an open access article distributed under the Creative Commons Attribution License, which permits unrestricted use, distribution, and reproduction in any medium, provided the original work is properly cited.

Iterative signal processing in communications has experienced an explosive rise in popularity in recent years. The catalytic origins of this paradigm-shifting new philosophy among communications experts can be traced to the invention of turbo coding, and the subsequent rediscovery of lowdensity parity check (LDPC) coding, both in the field of error control coding. Both systems rely on iterative decoding algorithms to achieve their astounding performance. However, iterative signal processing is not confined to the decoding of error control codes and rather quickly spread to many other possible applications. The purpose of this special issue is to examine the concept of iterative signal processing, highlight its potential, and draw the attention of communications engineers to this fascinating topic.

Of course, as the mathematicians know, but many communications experts may not realize, iterative processing has a long and productive history in the theory of linear matrix solution methods, that is, in finding approximative lowcomplexity numerical equation solving techniques which avoid the cubic complexity of direct solution methods. We start off this special issue with Axelsson's key paper giving an in-depth look at the methods and milestones in the development of linear iterative solution methods, to illustrate that iterative processing has long been a major tool in the areas of signal processing dealing with large systems of equations. The second paper by Huckle and Sedlack takes another detailed look and shows that it can be computationally efficient to replace classical smoothers used in iterative solution methods with sparse approximate inverses combined with subspace approximations. This technique can be applied to ill-posed problems such as recovering underlying information from blurred signals. The third paper by Donatelli and Serra-Capizzano discusses the use of antireflective boundary conditions for such deblurring problems. The precision of reconstruction and both iterative and noniterative regularization solvers are discussed.

The next three papers deal with the issue of separating signals in channels with large-scale crossinterference, where optimal, but exponentially complex, detection methods fail due to an infeasibility of implementation. These papers deal with situations where the number of mutually interfering signals is so large, that the traditional methods have to be replaced with something new, and this new methodology is based on iterative processing. Depending on the preferences of the authors, the iterative processing can be introduced as a variant of turbo decoding or a variant of iterative equation solution methods. Also popular is the view of the algorithm as a message-passing process on a connectivity graph-this approach was popularized by the LDPC coding community.

The first paper in this group by Schlegel et al., considers the problem of decoding signals that are modulated onto random signal waveforms, such as one may observe in multiple antenna fading channels. The authors show that an iterative cancellation decoder can achieve the capacity of this multiple access channel by exploiting signal redundancy introduced into the waveforms, and that this decoder has in fact the same order complexity as that of an iterative matrix solver. The second paper by Wo et al. addresses a very similar situation, called superposition modulation, where the individual waveforms are chosen deterministically, but correlated, and with signal attributes that shape the amplitude distribution of the signals. This is done to 
harness the shaping gain of $1.53 \mathrm{~dB}$ that theory promises with respect to standard one-dimensional pulse-amplitude modulation. The paper introduces and analyses an iterative receiver that overcomes the decoding complexity challenge. The third paper by Wo and Hoeher looks at the random waveform channel created by multiple antenna transmission systems and models the random mutual interference as correlated Gaussian noise. Rather than utilizing cancellation, the authors derive an iterative log-likelihood message passing algorithm based on this correlated Gaussian approximation and study examples where this decoder achieves the ideal performance of an interference-free system.

The next two papers consider specific complex signal processing challenges that are addressed by utilizing an iterative algorithm to obtain a solution with a low effort in complexity. The first paper by Kerr and Lodge treats the acquisition of an unknown phase of a spreading sequence. This problem is cast into the standard constraint form of a linear error control code. The received signal is then decoded iteratively by using a connectivity graph for that code model. The novelty lies in the fact that for each iteration a new code connectivity graph is formed using the outcomes of the previous iteration. The second paper by Ma et al., considers transmission of self-encoded signals over multiple antenna channels. The consequence of this self-encoding is that the received signal follows a finite-state model, and that the maximum-likelihood detector amounts to a sequence search with exponential complexity. It is precisely this complexity which is avoided by the use of an iterative algorithm.

The last paper by Psota et al. brings us back to error control coding, where iterative processing has made its first postclassical impact. In this paper, the authors discuss problems that can occur with the iterative decoding algorithm. They discuss the code structures that can cause the most common iterative decoders to get trapped in local incorrect solutions. A discussion of the issues and an illustration of the complexity of the problem are given using the example of LDPC codes, and an algorithmic enumeration procedure is presented.

Christian Schlegel

Peter Hoeher

Owe Axelsson

Lance Pérez 

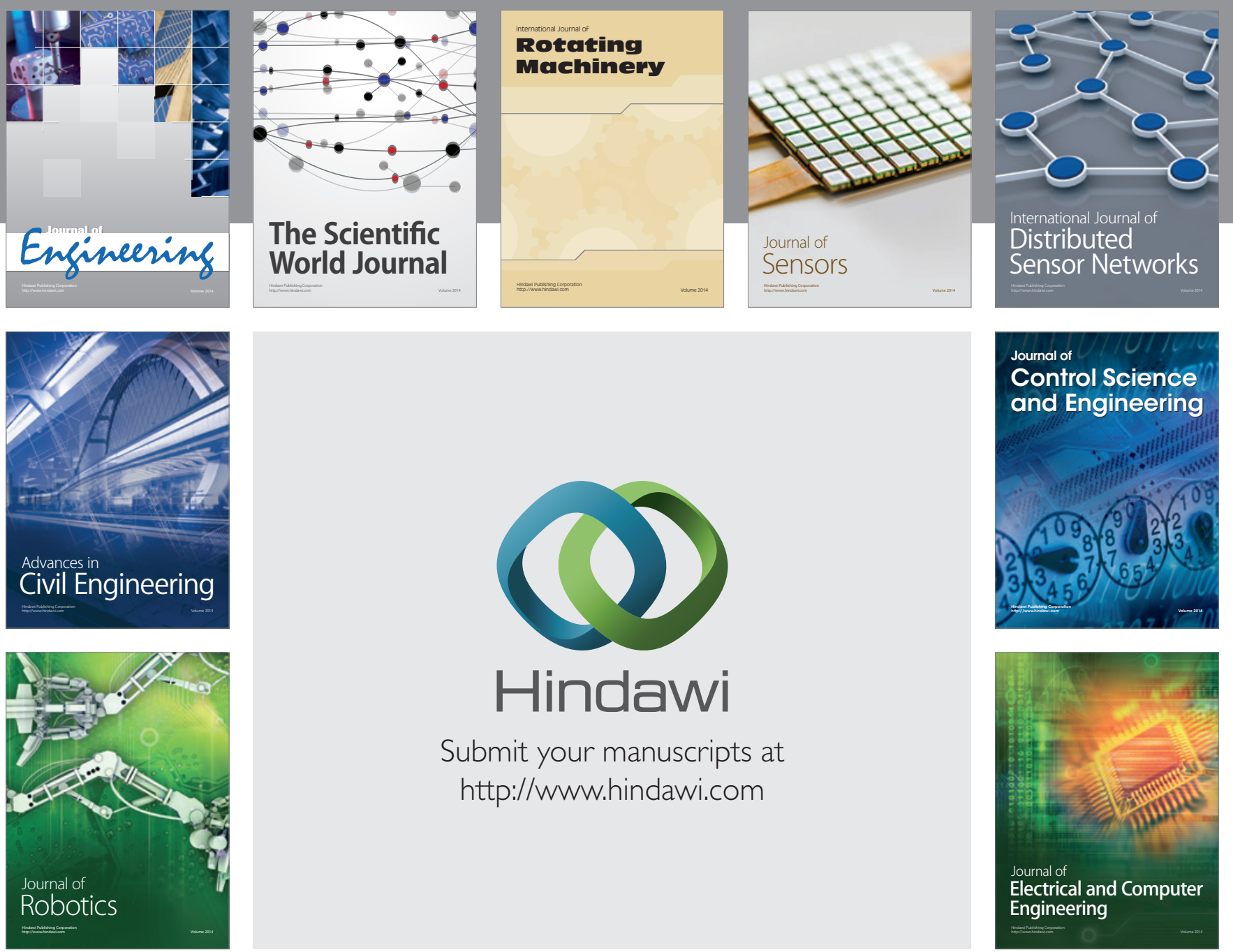

Submit your manuscripts at

http://www.hindawi.com
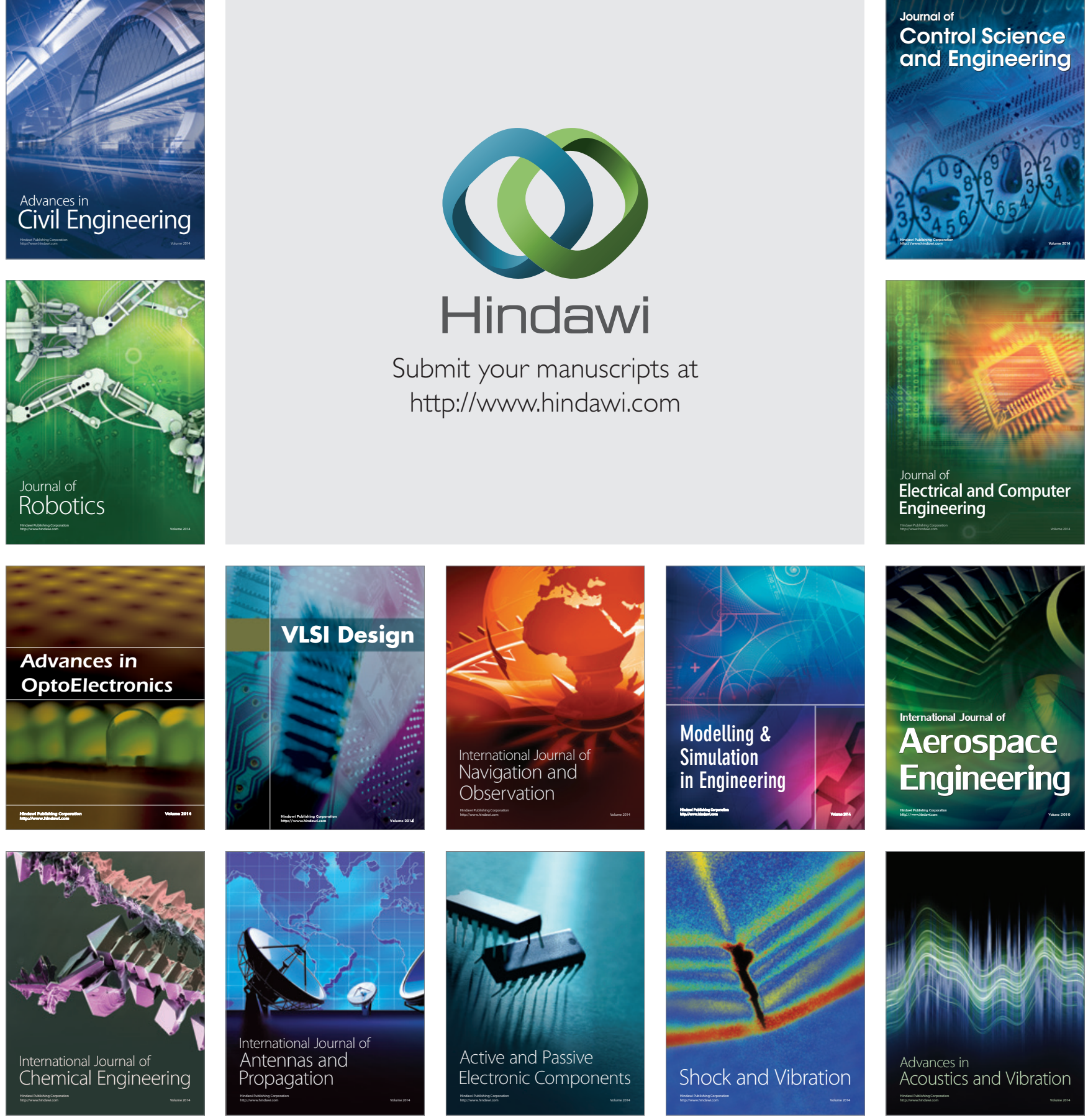